\title{
Smoldering subcutaneous polymicrobial infection concealed beneath a cast and a skin graft: Delayed wound healing due to recurring soft-tissue infections
}

\author{
Deepak Bharadia MD, Ashley Sturgeon MD, Daniel Baird MD, Jonathan Aldrete BA
}

\begin{abstract}
Injury to soft tissues secondary to trauma is common and may require surgical intervention depending on the extent of the injury and the structures involved. Motor vehicle accidents are a leading cause of traumatic injury and often require surgical irrigation and debridement to remove damaged and necrotic tissue while preserving tissue integrity and function. Surgical intervention carries its own risks, however, with the introduction of pathogens to the surgical site being a potential complication. This case of an 18-year-old woman with a chronic wound due to recurring skin and soft tissue infections (SSTIs) highlights the complications of surgical intervention and the difficulty of dealing with recurring SSTIs. The patient suffered a left knee injury with patellar tendon laceration secondary to trauma from a motor vehicle accident. She initially underwent surgical irrigation and debridement, followed by patellar tendon repair with a rotational flap to close the open wound. Following the initial closure in December 2019, the patient underwent six incision and drainage procedures over a 9-month period due to a chronic non-healing wound with recurring SSTIs. Despite these interventions, the patient ultimately underwent a total patellectomy due to recurring wound infections with penicillin resistant, methicillin-susceptible Staphylococcus aureus for which she was successfully treated with IV nafcillin over a period of 42 days.
\end{abstract}

Keywords: Chronic wound, skin and soft tissue infections, polymicrobial wounds

\section{INTRODUCTION}

The process of wound healing typically involves three stages: inflammatory, proliferative, and remodeling. In the case of traumatic injury, previously innocuous bacteria may gain entry to the wound site and disrupt the healing process, often resulting in the arrest of wound healing at the inflammatory stage, leading to a chronic wound. Skin and soft tissue infections can complicate wound healing and are responsible for many chronic wounds, which require further surgical

Corresponding author: Jonathan Aldrete

Contact Information: Jonathan.Aldrete@ttuhsc.edu

DOI: $10.12746 /$ swrccc.v9i37.745 debridement and closure and carry significant morbidity and financial burden to patients and the healthcare system. ${ }^{1,2}$ The pathogens responsible for chronic wound infections typically originate from the endogenous flora of a patient's skin or mucous membranes or hollow viscera, with the most commonly isolated pathogens being Staphylococcus aureus, Enterobacteriaceae, coagulase-negative Staphylococci, Enterococci, and Pseudomonas aeruginosa.

\section{CASE}

An 18-year-old woman sustained a left knee injury after being involved in a motor vehicle accident. She was ejected from her vehicle and sustained extensive soft tissue damage to her left knee and a patellar 


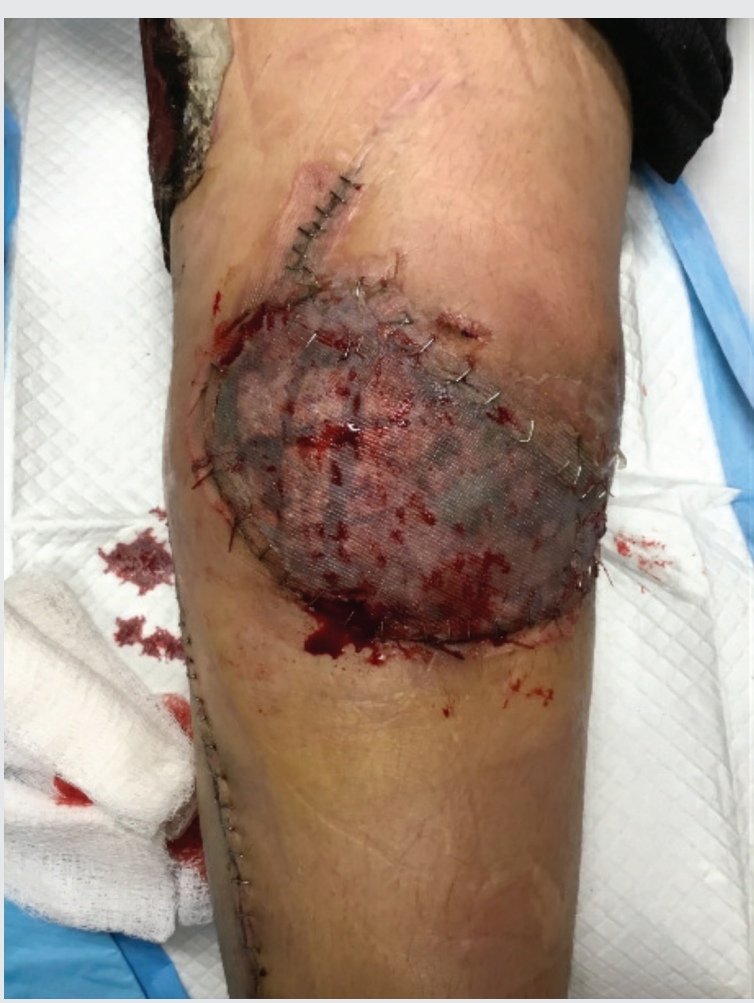

Figure 1. Split thickness graft to left knee with donor site to medial thigh at left lateral extremity $04 / 10 / 20$.

tendon laceration. She underwent incision and debridement (I\&D) followed by a rotational flap closure of the open wound. Following initial discharge on December 20, 2019, the patient returned to the Emergency Department (ED) on January 9, 2020, and was admitted for overnight observation due to suspected cellulitis vs. deep tissue infection. Vancomycin and piperacillin-tazobactam were started for empiric coverage and were later changed to trimethoprim-sulfamethoxazole (TMP-SMX). The patient returned to the ED and ultimately underwent another round of I\&D on February 10. She was again discharged with TMP-SMX and instructed to return to clinic in two weeks for wound inspection. On her return to the clinic, wound dehiscence was appreciated, and the patient underwent another round of I\&D on March 12 and was admitted for IV antibiotics. Cultures were positive for $S$. aureus sensitive to TMP-SMX, which the patient once again received after discharge.
Upon returning to the clinic two weeks later, a chronic wound with full thickness breakdown was appreciated, and the patient was admitted and underwent I\&D to the left joint with excision of subcutaneous fascia and muscle, medial gastrocnemius rotational flap, and split thickness graft to the left knee with donor site at the medial thigh (Figure 1). The patient was discharged and followed in the clinic until she returned to the ED on May 15, 2020, presenting with a medial thigh wound breakdown and necrosis (Figure 2). Intravenous vancomycin and piperacillin-tazobactam were administered, and she underwent necrotic tissue debridement on May 16 (Figure 3). A pathology report from a tissue specimen collected on May 16, 2020, demonstrated ulceration, dermal and pannicular neutrophilic abscesses, and necrosis with associated colonies of Gram-positive cocci, lipophagic fat necrosis, angioplasia, reactive vascular changes and fibrosis (Figures 4-5). Differential diagnoses for the observed pathology include bacterial abscess, cellulitis, superficial changes of necrotizing fasciitis and, more unlikely, pyoderma gangrenosum. While previous cultures had grown only $S$. aureus, cultures on May 26, 2020, grew Pseudomonas aeruginosa sensitive to ciprofloxacin and $S$. aureus sensitive to TMP-SMX. The patient was discharged on both antibiotics for two

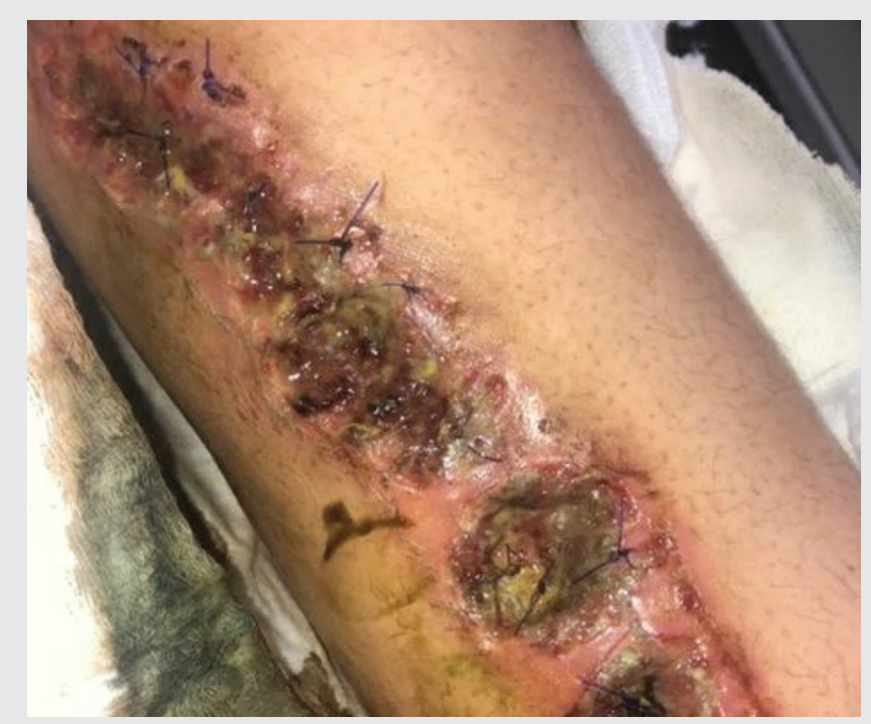

Figure 2. Patient presented to ED on $05 / 15 / 20$ with breakdown of medial calf incision with necrotic tissue. 


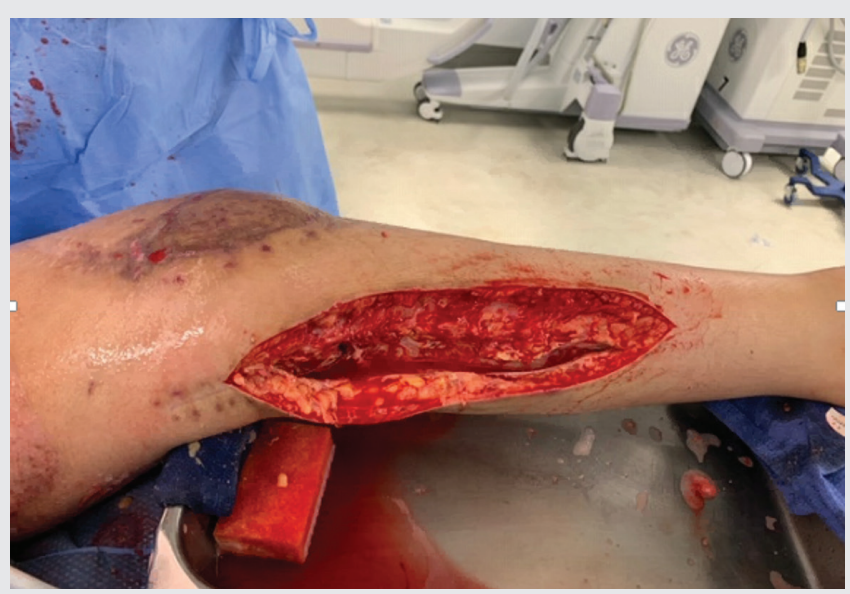

Figure 3. Wound after removal of necrotic tissue on 05-16-2020.

weeks but developed complaints of knee tenderness and pain soon after stopping the antibiotics. Over the course of four months, she subsequently underwent three more I\&D procedures, and, despite these interventions, ultimately underwent a total patellectomy on September 11, 2020. Tissue samples taken at the time grew penicillin-resistant, methicillin-susceptible $S$ aureus for which she was successfully treated with IV nafcillin over a period of 42 days.

\section{Discussion}

Chronic wounds are wounds that fail to heal over a period of 4-6 weeks. The etiology of chronic wounds is multifactorial; oxygenation, inflammatory processes, and infections all influence wound healing. ${ }^{3}$ Colonization of wounds by endogenous skin pathogens can arrest wound healing. While many of these pathogens are innocuous on the skin's surface, once they break the skin surface and gain access to soft tissues many become virulent. Traumatic injuries and surgical interventions often serve as the point of entry for these pathogens. ${ }^{4}$ Most of these infections are polymicrobial and often involve the development of biofilms, disrupting the healing process and complicating delivery of topical and systemic antibiotics., 5 The polymicrobial nature of these infections also complicates diagnosis and treatment; identifying the virulent pathogen in a polymicrobial infection is difficult and often results in inappropriate antimicrobial coverage that can delay treatment and drive antimicrobial resistance. ${ }^{7}$

Physicians have traditionally relied on culture media to identify the offending pathogen and to identify its susceptibilities to antimicrobial therapy. While these cultures often identify the pathogen correctly and guide treatment, their sensitivity and specificity are dependent on the type of swab done and its
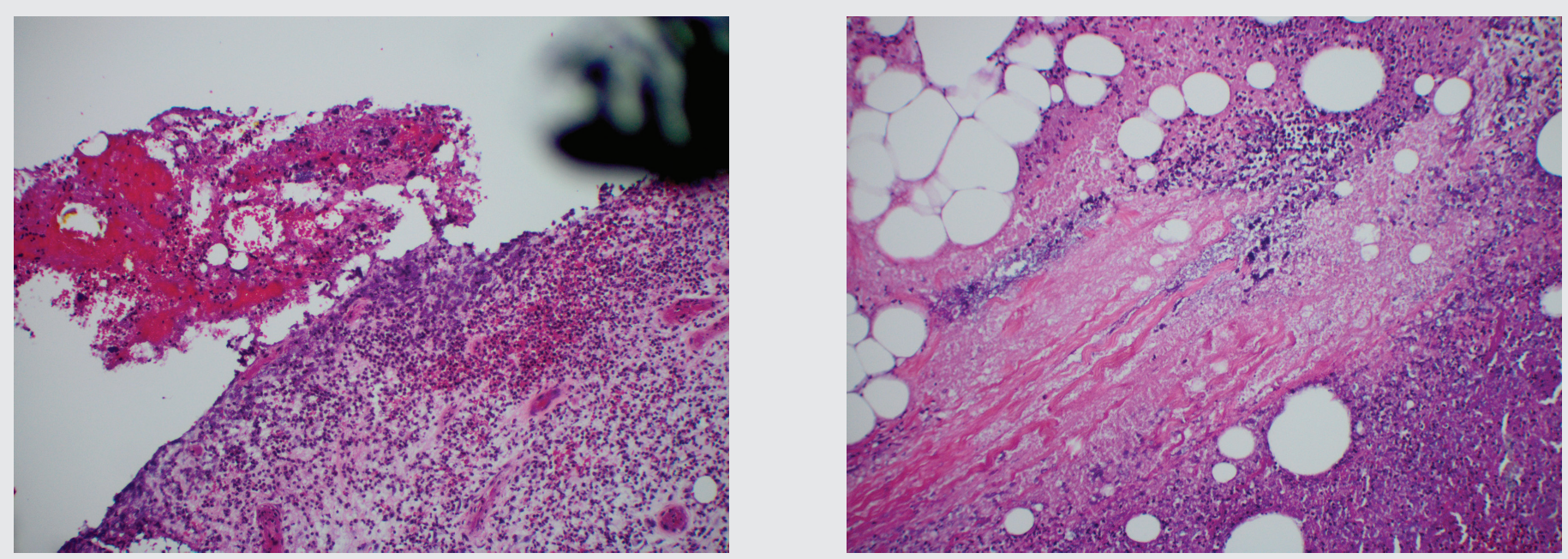

Figures 4 and 5. Pathology slides demonstrating ulceration, abscess formation, tissue necrosis, and liophagic fat necrosis. 
location. Superficial swabs are not as accurate as deep tissue cultures, and often miss the relevant pathogen. ${ }^{8}$ Treatment efficacy is contingent on the prompt identification of the infectious pathogens and their susceptibility, but treatment outcome is not always guaranteed. ${ }^{9}$ The extent of injury to the anatomic structures and soft tissues may be such that vascular and lymphatic drainage is compromised, creating an excellent environment for recurrent infections.

With an estimated incidence of $2-5 \%$ in patients undergoing inpatient surgery, SSTIs place great financial and personal burden on patients and hospitals. ${ }^{10}$ While there are certain risk factors associated with the development of chronic wounds (extent of injury, age, diabetes, smoking status, vasculopathies, etc,) our patient had few identifiable risk factors (traumatic injury, occasional consumption of marijuana). This case demonstrates that despite sound clinical judgement and appropriate surgical interventions, culturing techniques, and appropriate antimicrobial coverage for suspected pathogens, chronic wounds secondary to traumatic injury often fail to heal, and may require further surgical revisions, even amputations, to resolve. ${ }^{10}$

Article citation: Bharadia D, Sturgeon A, Baird D, Aldrete J. Smoldering subcutaneous polymicrobial infection concealed beneath a cast and a skin graft: delayed wound healing due to recurring soft-tissue infections. The Southwest Respiratory and Critical Care Chronicles 2021;9(37):78-81

From: Department of Dermatology, Texas Tech University Health Sciences Center, Lubbock, Texas

Submitted: $8 / 10 / 2020$

Accepted: $1 / 18 / 2021$

Reviewer: Jacob Nichols MD

Conflicts of interest: none

This work is licensed under a Creative Commons

Attribution-ShareAlike 4.0 International License.

\section{REFERENCES}

1. McCosker L, Tulleners R, Cheng Q, et al. Chronic wounds in Australia: a systematic review of key epidemiological and clinical parameters. Int Wound Journal 2019;16:84-95.

2. Olsson M, Järbrink K, Divakar U, et al. The humanistic and economic burden of chronic wounds: a systematic review. Wound Rep Reg 2019;27:114-125.

3. Bowler PG. Wound pathophysiology, infection, and therapeutic options. Annals Med 2002;34(6):419-427.

4. Landis S. Chronic wound infection and antimicrobial use. Adv Skin Wound Care 2008;21(11):531-540.

5. Gompelman M, van Asten S, Peters E. Update on the role of infection and biofilms in wound healing: pathophysiology and treatment. Plast Reconstr Surg 2016;138(3S):61S-70S.

6. Johnson T, Gómez B, McIntyre M, Dubick M, et al. The cutaneous microbiome and wounds: new molecular targets to promote wound healing. Int J Mol Sci 2018;19:2699.

7. Rahim K, Saleha S, Zhu X, Huo L, et al. Bacterial contribution in chronicity of wounds. Microb Ecol 2017;73(3):710-721.

8. Chakraborti C. Sensitivity of superficial cultures in lower extremity wounds. J Hospital Med 2010;5(7).

9. Siddiqui, Abdul R., Bernstein, Jack M. Chronic wound infection: facts and controversies. Clinics in Dermatology September-October 2010;28(5):519-526.

10. Ban $\mathrm{K}$, Minei J, Laronga $\mathrm{C}$, Brian G, et al. American College of Surgeons and Surgical Infection Society: Surgical Site Infection Guidelines, 2016 Update, J Amer College Surgeons 2017;224(1):59-74. 